\title{
Real-Time Link-Reliability Routing for QoS in Wireless Sensor Networks
}

\author{
Shiva Prakash T ${ }^{1}$, Raja K B ${ }^{1}$, Venugopal K R ${ }^{1}$, S S Iyengar ${ }^{2}$, L M Patnaik ${ }^{3}$ \\ ${ }^{1}$ (University Visvesvaraya College of Engineering, India) \\ ${ }^{2}$ (Florida International University, USA) \\ ${ }^{3}$ (Indian Institute of Science, India)
}

\begin{abstract}
This paper proposes a Real-Time Link Reliability Routing protocol for wireless sensor networks (WSNs). The protocol achieves to reduce packet deadline miss ratio while considering link reliability, two-hop velocity and power efficiency and utilizes memory and computational effective methods for estimating the link metrics. Numerical results provide insights that the protocol has a lower packet deadline miss ratio and improved sensor network lifetime. The results show that the proposed protocol is a feasible solution to the QoS routing problem in wireless sensor networks that support real-time applications.
\end{abstract}

Keywords: Deadline Miss Ratio (DMR), energy efficiency, link reliability, Quality-of-service (QoS), two-hop neighbors, Wireless Sensor Networks (WSNs).

\section{INTRODUCTION}

Wireless Sensor Networks (WSNs) form a framework to accumulate and analyze real time data in smart environment applications. WSNs are composed of inexpensive low-powered micro sensing devices called motes [1], having limited computational capability, memory size, radio transmission range and energy supply. Sensors are spread in an environment without any predetermined infrastructure and cooperate to accomplish common monitoring tasks which usually involve sensing environmental data. With WSNs, it is possible to assimilate a variety of physical and environmental information in near real time from inaccessible and hostile locations.

WSNs have a wide variety of applications in military, industry, environment monitoring and health care. WSNs operate unattended in harsh environments, such as border protection and battlefield reconnaissance hence help to minimize the risk to human life. WSNs are used extensively in the industry for factory automation, process control, real-time monitoring of machines, detection of radiation and leakages and remote monitoring of contaminated areas, aid in detecting possible system deterioration and to initiate precautionary maintenance routine before total system breakdown. WSNs are being rapidly deployed in patient health monitoring in a hospital environment, where different health parameters are obtained and forwarded to health care servers accessible by medical staff and surgical implants of sensors can also help monitor the health of patients.

Emerging WSNs have a set of stringent QoS requirements that include timeliness, high reliability, availability and integrity. The competence of a WSN lies in its ability to provide these QoS requirements. The timeliness and reliability level for data exchanged between sensors and control station is of paramount importance especially in real time scenarios. The deadline miss ratio (DMR) [6], defined as the ratio of packets that cannot meet the deadlines should be minimized. Sensor nodes typically use batteries for energy supply. Hence, energy efficiency and load balancing form important objectives while designing protocols for WSNs. Therefore, providing corresponding QoS in such scenarios pose to be a great challenge. Our proposed protocol is motivated primarily by the deficiencies of the previous works (explained in the Section 2) and aims to provide better Quality of Service.

This paper explores the idea of incorporating QoS parameters in making routing decisions i.e.,: (i) reliability (ii) latency and (iii) energy efficiency. Traffic should be delivered with reliability and within a deadline. Furthermore, energy efficiency is intertwined with the protocol to achieve a longer network lifetime. Hence, the protocol is named, Real-time Link Reliability Routing (RTLRR). The protocol proposes the following features.

1. Link reliability is considered while choosing the next router; this selects paths which have higher probability of successful delivery.

2. Routing decision is based on two-hop neighborhood information and dynamic velocity that can be modified according to the required deadline, this results in significant reduction in end-to-end DMR (deadline miss ratio).

3. Choosing nodes with higher residual energy balances the load among nodes and results in prolonged lifetime of the network. 
Real-Time Link-Reliability Routing for QoS in Wireless Sensor Networks

Table 1: Our results and comparison with previous results for QoS routing in Wireless Sensor Networks

\begin{tabular}{|l|l|l|l|l|}
\hline \multicolumn{1}{|c|}{$\begin{array}{l}\text { Related } \\
\text { Work }\end{array}$} & Protocol Name & Considered Metric & Estimation Method & Performance \\
\hline $\begin{array}{l}\text { Tian He et } \\
\text { al., [2] }\end{array}$ & $\begin{array}{l}\text { SPEED (Stateless } \\
\text { Protocol for End-to- } \\
\text { End Delay) }\end{array}$ & $\begin{array}{l}\text { One-hop delay and } \\
\text { residual energy }\end{array}$ & $\begin{array}{l}\text { EWMA (Exponential } \\
\text { Weighted Moving } \\
\text { Average) }\end{array}$ & $\begin{array}{l}\text { Improves end-to-end delay and provides } \\
\text { good response to congestion }\end{array}$ \\
\hline $\begin{array}{l}\text { E. Felemban } \\
\text { et al., [3] }\end{array}$ & $\begin{array}{l}\text { MMSPEED (Multi- } \\
\text { path and Multi-SPEED } \\
\text { Routing Protocol) }\end{array}$ & $\begin{array}{l}\text { One-hop delay, link } \\
\text { reliability and } \\
\text { residual energy }\end{array}$ & $\begin{array}{l}\text { EWMA (Exponential } \\
\text { Weighted Moving } \\
\text { Average) }\end{array}$ & $\begin{array}{l}\text { Provides service differentiation and } \\
\text { probabilistic QoS guarantee in the } \\
\text { timeliness and reliability domains. }\end{array}$ \\
\hline $\begin{array}{l}\text { Chipera et } \\
\text { al.,[4] }\end{array}$ & $\begin{array}{l}\text { RPAP (Real-Time } \\
\text { Power Aware Routing) }\end{array}$ & $\begin{array}{l}\text { One-hop delay and } \\
\text { transmission power }\end{array}$ & Jacobson Algorithm & $\begin{array}{l}\text { Provides real-time routing and dynamic } \\
\text { power adaptation to achieve application } \\
\text { specific communication delays at low } \\
\text { energy cost. }\end{array}$ \\
\hline $\begin{array}{l}\text { Y. Li et } \\
\text { al.,[5] }\end{array}$ & $\begin{array}{l}\text { THVR (Two-Hop } \\
\text { Velocity Based }\end{array}$ & $\begin{array}{l}\text { Two-hop delay and } \\
\text { Residual energy }\end{array}$ & $\begin{array}{l}\text { WMEWMA (Window } \\
\text { Mean Exponential } \\
\text { Weighted Moving } \\
\text { Average) }\end{array}$ & $\begin{array}{l}\text { Routing Decision is made based on two- } \\
\text { hop velocity integrated with energy } \\
\text { balancing mechanism which achieves } \\
\text { lower end-to-end DMR and higher } \\
\text { energy utilization efficiency. }\end{array}$ \\
\hline This Paper & $\begin{array}{l}\text { RTLRR (Real-Time } \\
\text { Link Reliability and } \\
\text { residual energy }\end{array}$ & $\begin{array}{l}\text { Two-hop delay, link } \\
\text { reliability and } \\
\text { residual energy }\end{array}$ & EWMA and WMEWMA & $\begin{array}{l}\text { The protocol considers link reliability } \\
\text { and uses dynamic velocity as per the } \\
\text { desired deadline, energy is efficiently } \\
\text { balanced among the nodes. }\end{array}$ \\
\hline
\end{tabular}

The proposed protocol is devised using a modular design; separate modules are dedicated to each QoS requirement. The link reliability estimation and link delay estimation modules use memory and computational effective methods suitable for WSNs. The node forwarding module is able to make the optimal routing decision using the estimated metrics.

We test the performance of our proposed approaches by implementing our algorithms using ns-2 simulator. Our results demonstrate the performance and benefits of RTLRR over earlier algorithms.

The rest of the paper is organized as follows: Section 2 gives a review of the Related Works. Section 3 and Section 4 explains the Problem and the Network Model, assumptions. The working of the algorithm is explained in Section 5. Section 6 is devoted to the Simulation and Evaluation of the algorithm. Conclusions are presented in Section 7.

\section{RELATED WORK}

Stateless routing protocols which do not maintain per-route state is a favorable approach for WSNs. The idea of stateless routing is to use location information available to a node locally for routing, i.e., the location of its own and that of its one-hop neighbors without the knowledge about the entire network. These protocols scale well in terms of routing overhead because the tracked routing information does not grow with the network size or the number of active sinks. Parameters like distance to sink, energy efficiency and data aggregation, need to be considered to select the next router among the one-hop neighbors.

SPEED (Stateless Protocol for End-to-End Delay) [2] is a well known stateless routing protocol for real-time communication in sensor networks. It is based on geometric routing protocols such as greedy forwarding GPSR (Greedy Perimeter State Routing) [7] [8]. It uses non-deterministic forwarding to balance each flow among multiple concurrent routes. SPEED combines Medium Access Control (MAC) and network layer mechanism to maintain a uniform speed across the network, such that the delay a packet experiences is directly proportional to its distance to the sink. At the MAC layer, a single hop relay speed is maintained by controlling the drop/relay action in a neighbor feedback loop. Geographic forwarding is used to route data to its destination selecting the next hop as a neighbor from the set of those with a relay speed higher that the desired speed. A back pressure re-routing mechanism is employed to re-route traffic around congested areas if necessary. Lu et al., [9] describe a packet scheduling policy, called Velocity Monotonic Scheduling, which inherently accounts for both time and distance constraints. Sequential Assignment Routing (SAR) [10] is the first routing protocol for sensor networks that creates multiple trees routed from one-hop neighbors of the sink by taking into consideration both energy resources, QoS metric on each path and priority level of each packet. However, the protocol suffers from the overhead of maintaining the tables and states at each sensor node especially when the number of nodes is large.

MMSPEED (Multi-path and Multi-SPEED Routing Protocol) [3] is an extension of SPEED that focuses on differentiated QoS options for real-time applications with multiple different deadlines. It provides differentiated QoS options both in timeliness domain and the reliability domain. For timeliness, multiple QoS levels are supported by providing multiple data delivery speed options. For reliability, multiple reliability requirements are supported by probabilistic multi-path forwarding. The protocol provides end-to-end QoS provisioning by employing localized geographic forwarding using immediate neighbor information without endto-end path discovery and maintenance. It utilizes dynamic compensation which compensates for inaccuracy of 
local decision as a packet travels towards its destination. The protocol adapts to network dynamics. MMSPEED does not include energy metric during QoS route selection. Chipera et al., [4] (RPAR: Real-Time Power Aware Routing) have proposed another variant of SPEED. Where a node will change its transmission power by the progress towards destination and packet's slack time in order to meet the required velocity; they have not considered residual energy and reliability.

Mahapatra et al., [11] assign an urgency factor to every packet depending on the residual distance and time the packet needs to travel, and determines the distance the packet needs to be forwarded closer to the destination to meet its deadline. Multi-path routing is performed only at the source node for increasing reliability. Some routing protocols with congestion awareness have been proposed in [12] [13]. Other geographic routing protocols such as [14] [15] [16] [17] deal only with energy efficiency and transmission power in determining the next router. Seada et al., [28] proposed the PRR (Packet Reception Rate) $\times$ Distance greedy forwarding that selects the next forwarding node by multiplying the PRR by the distance to the destination. Recent geographical routing protocols have been proposed, such as DARA (Distributed Aggregate Routing Algorithm) [19], GREES (Geographic Routing with Environmental Energy Supply) [20], DHGR (Dynamic Hybrid Geographical Routing) [21], and EAGFS (Energy Aware Geographical Forwarding Scheme) [22]. They define either the same combined metric (of all the considered QoS metrics) [2], [22], [20], or several services but with respect to only one metric [4] [3].

Sharif et al., [23] presented a new transport layer protocol that prioritizes sensed information based on its nature while simultaneously supporting the data reliability and congestion control features. Rusli et al., [24] propose an analytical framework model based on Markov Chain of OR and M/D/1/K queue to measure its performance in term of end-to-end delay and reliability in WSNs. Koulali et al., [25] propose a hybrid QoS routing protocol for wireless sensor networks based on a customized Distributed Genetic Algorithm (DGA) that accounts for delay and energy constraints. Yunbo Wang et al., [26] investigate the end-to-end delay distribution, they develop a comprehensive cross-layer analysis framework, which employs a stochastic queuing model in realistic channel environments. Ehsan et al., [27] propose energy and cross-layer aware routing schemes for multichannel access WSNs that account for radio, MAC contention, and network constraints.

All the above routing protocols are based on one-hop neighborhood information. However, it is expected that multi-hop information can lead to improved performance in many issues including message broadcasting and routing. Spohn et al., [28] propose a localized algorithm for computing two-hop connected dominating set to reduce the number of redundant broadcast transmissions. An analysis in [29] shows that in a network of $n$ nodes of total of $O(n)$ messages are required to obtain 2-hop neighborhood information and each message has $O(\log n)$ bits. Chen et al., [30] study the performance of 1-hop, 2-hop and 3-hop neighborhood information based routing and propose that gain from 2-hop to 3-hop is relatively minimal, while that from 1hop to 2-hop based routing is significant.

Li et al., [5] have proposed a Two-Hop Velocity Based Routing Protocol (THVR). The routing choice is decided on the two-hop relay velocity and residual energy, an energy efficient packet drop control is included to enhance packet utilization efficiency while keeping low packet deadline miss ratio. However, THVR does not consider reliability while deciding the route. The protocol proposed in this paper is different from THVR. It considers reliability and uses dynamic velocity that can be altered for each packet as per the desired deadline. It considers energy efficiently and balances the load only among nodes estimated to offer the required QoS.

\section{PROBLEM DEFINITION}

The topology of a wireless sensor network may be described by a graph $G=(N, L)$, where $N$ is the set of nodes and $\mathrm{L}$ is the set of links. The objectives are to,

- Minimize the deadline miss ratio (DMR).

- $\quad$ Reduce the end-to-end packet delay.

- Improve the energy efficiency (ECPP-Energy Consumed Per Packet) of the network.

\section{NETWORK MODEL AND ASSUMPTIONS}

In our network model, we assume the following:

- The wireless sensor nodes consists of $\mathrm{N}$ sensor nodes and a sink, the sensors are distributed randomly in a field.

- The nodes are aware of their positions through internal global positioning system (GPS), so each sensor has an estimate of its current position.

- The $\mathrm{N}$ sensor nodes are powered by a non renewable on board energy source. When this energy supply is exhausted the sensor becomes non-operational. All nodes are supposed to be aware of their residual energy and have the same transmission power range.

- The sensors share the same wireless medium each packet is transmitted as a local broadcast in the neighborhood. The sensors are neighbors if they are in the transmission range of each other and can 
directly communicate with each other. We assume a MAC protocol, i.e., IEEE 802.11 which ensures that among the neighbors in the local broadcast range, only the intended receiver keeps the packet and the other neighbors discard the packet.

- Like all localization techniques, [2] [3] [31] [32] [33] each node needs to be aware of its neighboring nodes current state (ID, position, link reliability, residual energy etc), this is done via HELLO messages.

- Nodes are assumed to be stationary or having low mobility, else additional HELLO messages will be needed to keep the nodes up-to-date about the neighbor nodes.

- In addition, each node sends a second set of HELLO messages to all its neighbors informing them about its one-hop neighbors. Hence, each node is aware of its one-hop and two-hop neighbors and their current state.

- The network density is assumed to be high enough to prevent the void situation. To ensure a high-quality product, diagrams and lettering MUST be either computer-drafted or drawn using India ink.

\section{Algorithm}

RTLRR has three components: a link reliability estimator, a delay estimator, a node forwarding metric incorporated with the dynamic velocity assignment policy. The proposed protocol RTLRR implements the modules for estimating transmission delay and packet delivery ratios using efficient methods. The packet delay is estimated at the node itself and the packet delivery ratio is estimated by the neighboring nodes. These parameters are updated on reception of a HELLO packet; the HELLO messages are periodically broadcast to update the estimation parameters. The overhead caused by the 1-hop and 2-hop updating are reduced by piggybacking the information in ACK, hence improving the energy efficiency. The protocol is based on the following parameters: (i) Link Reliability Estimation; (ii) Link Delay Estimation; and (iii) Node Forwarding Metric.

\section{Link Reliability Estimation}

The Packet Reception Ratio (PRR) of the link relaying node $\mathrm{x}$ to $\mathrm{y}$ is denoted by $\mathrm{prr}_{\mathrm{xy}}$. It denotes the probability of successful delivery over the link. Window Mean Exponential Weighted Moving Average based link quality estimation is used for the proposed protocol. The window mean exponential weighted moving average estimation applies filtering on PRR, thus providing a metric that resists transient fluctuations of PRR, yet is responsive to major link quality changes. This parameter is updated by node $y$ at each window and inserted into the HELLO message packet for usage by node $x$ in the next window.

Equation 1 shows the window mean exponential weighted moving average estimation of the link reliability, $r$ is the number of packets received, $m$ is the number of packets missed and $\alpha \in[0,1]$ is the history control factor, which controls the effect of the previously estimated value on the new one, $r /(r+m)$ is the newly measured PRR value.

$$
p r r_{x y}=\alpha \times p r r_{x y}+(1-\alpha) \times \frac{r}{r+m}
$$

The PRR estimator is updated at the receiver side for each $w$ (window size) received packets, the computation complexity of this estimator is $O(1)$. The appropriate values for $\alpha$ and $w$ for a stable window mean exponential weighted moving average are $w=30$ and $\alpha=0.5$ [34].

\section{Link Reliability Estimation}

The delay indicates the time spent to send a packet from node $x$ to its neighbor $y$, it is comprised of the queuing delay $\left(\right.$ delay $\left._{\mathrm{Q}}\right)$, contention delay $\left(\right.$ delay $\left._{\mathrm{C}}\right)$ and the transmission delay $\left(\right.$ delay $\left._{\mathrm{T}}\right)$.

$$
\text { delay }_{x y}=\text { delay }_{Q}+\text { delay }_{C}+\text { delay }_{T}
$$

If $\mathrm{t}_{\mathrm{s}}$ is the time the packet is ready for transmission and becomes head of transmission queue, $\mathrm{t}_{\mathrm{ack}}$ the time of the reception of acknowledgment, $\mathrm{BW}$ the network bandwidth and size of the acknowledgment then, $t_{a c k}$ sizeof $(\mathrm{ACK}) / \mathrm{BW}-\mathrm{t}_{\mathrm{s}}$ is the recently estimated delay and $\beta \epsilon[0,1]$ is the tunable weighting coefficient. Equation 3 shows the EWMA (Exponential Weighted Moving Average) update for delay estimation, which has the advantage of being simple and less resource demanding.

$$
\text { delay }_{x y}=\beta \times \text { delay }_{x y}+(1-\beta) \times\left(t_{a c k}-\operatorname{sizeof}(A C K) / B W-t_{s}\right)
$$

delay $_{x y}$ includes estimation of the time interval from the packet that becomes head of line of $x$ 's transmission queue until its reception at node $y$. This takes into account all delays due to contention, channel sensing, channel reservation (RTS/CTS) if any, depending on the medium access control (MAC) protocol, time slots etc. The computation complexity of this estimator is $O(1)$. The delay information is further exchanged among two-hop neighbors. 


\section{Node Forwarding Metric}

In the wireless sensor network, described by a graph $G=(N, L)$. If node $x$ can transmit a message directly to node $y$, the ordered pair is an element of $L$. We define for each node $x$ the set $\mathrm{N}_{1}(\mathrm{x})$, which contains the nodes in the network $G$ that are one-hop i.e., direct neighbors of $x$.

$$
N_{1}(x)=\{y:(x ; y) \in E, y \neq x\}
$$

Likewise, the two-hop neighbors of $\mathrm{x}$ is the set $\mathrm{N}_{2}(\mathrm{x})$ i.e.,

$$
N_{2}(x)=\left\{z:(y ; z) \in E, y \in N_{1}(x), z \neq x\right\}
$$

The Euclidean distance between a pair of nodes $\mathrm{x}$ and $\mathrm{y}$ is defined by $\operatorname{dist}(\mathrm{x}, \mathrm{y})$. We define $F_{1}^{+p}(x)$ as the set of $\mathrm{x}$ 's one-hop favorable forwarders providing positive progress towards the destination $\mathrm{D}$. It consists of nodes that are closer to the destination than $x$, i.e.,

$$
F_{1}^{+p}(x)=\left\{y \in N_{1}(x): \operatorname{dist}(x, D)-\operatorname{dist}(y, D)>0\right\}
$$

$F_{2}^{+p}(x)$ is defined as the set of two-hop favorable forwarders i.e.,

$$
F_{2}^{+p}(x)=\left\{y \in F_{1}^{+p}(x), z \in N_{1}(y): \operatorname{dist}(y, D)-\operatorname{dist}(z, D)>0\right\}
$$

Table 2: Algorithm: Real-Time Link Reliability Routing (RTLRR)

$$
\begin{aligned}
& \text { Input : } x, D, F_{1}^{+p}(x), F_{2}^{+p}(x), l t \\
& \text { Output }: \text { Node }: y \\
& V_{\text {req }}=\frac{\operatorname{dist}(x, D)}{l t} ; \\
& \text { For each } y \in F_{2}^{+p}(x) \text { do } \\
& V_{x y \rightarrow z}=\frac{\operatorname{dist}(x, D)-\operatorname{dist}(z, D)}{\operatorname{delay} y_{x y}+\operatorname{delay}_{y z}} ; \\
& \quad S_{\text {req }}=\left\{F_{2}^{+p}(x): V_{x y \rightarrow z} \geq V_{\text {req }}\right\} ; \\
& \text { If }\left(\mid S_{\text {req }}\right)=1 \text { then } \\
& \text { Return } y \in S_{\text {req }} ; \\
& \text { Else } \quad \text { For each } y \in S_{\text {req }} \text { do } \\
& \quad r v e_{x y \rightarrow z}=A \times \frac{p r r_{x y}}{\sum_{y \in S_{r e q}}\left(p r r_{x y}\right)}+B \times \frac{V_{x y \rightarrow z}}{\sum_{y \in S_{r e q}} V_{x y \rightarrow z}} \\
& \quad+C \times \frac{E_{y} / E_{y}^{0}}{\sum_{y \in S_{r e q}}\left(E_{y} / E_{y}^{0}\right)} ; \\
& \text { Find } y \text { with } M a x\left\{r v e_{x y \rightarrow z}\right\} ; \\
& \text { Return } y \in S_{r e q} ;
\end{aligned}
$$

We define two velocities; the required velocity $\mathrm{V}_{\text {req }}$ and the velocity offered by the two-hop favorable forwarding pairs. In SPEED, the velocity provided by each of the forwarding nodes in $\left(F_{1}^{+p}(x)\right)$ is.

$$
V_{x y}=\frac{\operatorname{dist}(x, D)-\operatorname{dist}(y, D)}{\operatorname{delay}_{x y}}
$$

As in THVR, by two-hop knowledge, node $x$ can calculate the velocity offered by each of the two-hop favorable forwarding pairs $\left(F_{1}^{+p}(x), F_{2}^{+p}(x)\right)$ i.e., 


$$
V_{x y \rightarrow z}=\frac{\operatorname{dist}(x, D)-\operatorname{dist}(z, D)}{\operatorname{delay}_{x y}+\operatorname{delay}_{y z}}
$$

Where, $\mathrm{y} \in F_{1}^{+p}(x)$ and $\mathrm{z} \in F_{2}^{+p}(x)$. The required velocity is relative to the progress made towards the destination [4] and the time remaining to the deadline, lt (lag time). The lag time is the time remaining until the packet deadline expires. At each hop, the transmitter renews this parameter in the packet header i.e.,

$$
l t=l t_{p}-\left(t_{t x}-t_{r x}+\operatorname{sizeof}(\text { packet }) / B W\right)
$$

Where $l t$ is the time remaining to the deadline $\left(t_{\text {req }}\right)$, $l t_{\mathrm{p}}$ is the previous value of $l t,\left(t_{t x}-t_{r x}+\right.$ sizeof(packet)/BW) accounts for the delay from reception of the packet until transmission. On reception of the packet the node $x$, uses $l t$ to calculate the required velocity $\mathrm{V}_{\text {req }}$ for all nodes in $\left(F_{1}^{+p}(x), F_{2}^{+p}(x)\right)$ as show in Equation 11.

$$
V_{\text {req }}=\frac{\operatorname{dist}(x, D)}{l t}
$$

The node pairs satisfying $V_{x y \rightarrow z} \geq V_{\text {req }}$ form the set of nodes $\mathrm{S}_{\text {req. }}$. For the set $\mathrm{S}_{\text {req }}$ we calculate the shared metric $r v e_{x y \rightarrow z}$, incorporating the node's link reliability, velocity towards destination and remaining energy level of neighbors in $S_{\text {req, }}$ as show in Equation 12.

$$
r v e_{x y \rightarrow z}=A \times \frac{p r r_{x y}}{\sum_{y \in S_{\text {req }}}\left(p r r_{x y}\right)}+B \times \frac{V_{x y \rightarrow z}}{\sum_{y \in S_{\text {req }}} V_{x y \rightarrow z}}+C \times \frac{E_{y} / E_{y}^{0}}{\sum_{y \in S_{\text {req }}}\left(E_{y} / E_{y}^{0}\right)}
$$

$\mathrm{A}, \mathrm{B}$ and $\mathrm{C}$ are the weighting factors for combining reliability, velocity and energy into the shared metric $(A+B+C=1)$. The node $y$ with the largest $r v e_{x y \rightarrow z}$ will be chosen as the forwarder and the process continuous till the destination is reached. The Real-time Link Reliability Routing Protocol is shown in Table 2, the computation complexity of this algorithm is $O\left(F_{2}^{+p}(x)\right)$. Our proposed protocol is different from THVR, as it considers reliability and dynamic velocity that can be adjusted for each packet according to the required deadline. It balances the load only among nodes estimated to offer the required QoS.

\section{RTLRR: An Example}

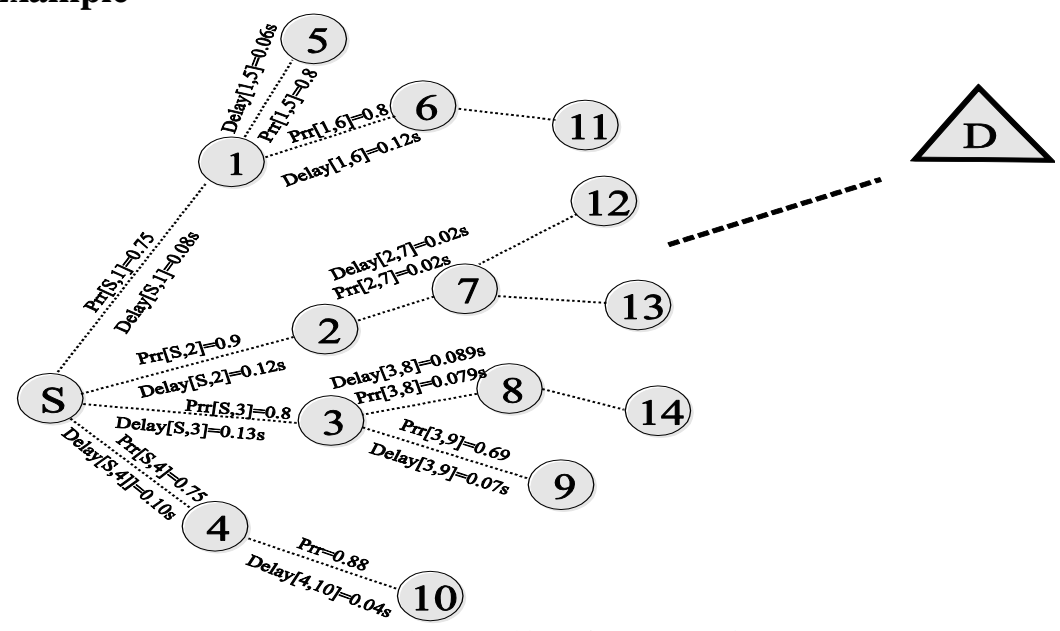

Fig. 1. A Study on working of RTLR Routing.

We bring out the working for the proposed protocol in a case study. From Fig.1 if a packet is to be sent from S to $\mathrm{D}$, then nodes $\{1,2,3,4\} \in F_{1}^{+p}(S) .\{5,6\} \in F_{1}^{+p}(1),\{7\} \in F_{1}^{+p}(2),\{10\} \in F_{1}^{+p}(4),\{14\} \in$ $F_{1}^{+p}(8),\{12,13\} \in F_{7}^{+p}(7),\{7\} \in F_{1}^{+p}(2)$. The distance between the various nodes and the destination are: $\quad(S, D)=150 m, \quad(1, D)=120 m, \quad(2, D)=108 m, \quad(3, D)=114 m, \quad(4, D)=127.5 m$, $(5, D)=117 m, \quad(6, D)=97.5 m, \quad(7, D)=110 m, \quad(8, D)=90 m, \quad(9, D)=97.5 m \quad$ and 
$(10, D)=117 m$.

Let the required velocity,

$$
V_{\text {req }}=\frac{150 \mathrm{~m}}{0.55 \mathrm{~s}}=272.7 \mathrm{~m} / \mathrm{s}
$$

Here, the end-to-end deadline is $0.55 \mathrm{~s}$. By, Eqn. 8 , each node calculates the velocity $\left(V_{x y}\right)$ provided by each of its forwarding nodes in $F_{1}^{+p}(S)$,

$$
V_{S 1}=\frac{150 m-120 m}{0.08 s}=375 m / s
$$

Likewise, the velocity provided by $\mathrm{V}_{\mathrm{S} 2}=(150 \mathrm{~m}-108 \mathrm{~m}) / 0.12 \mathrm{~s}=350 \mathrm{~m} / \mathrm{s}, \mathrm{V}_{\mathrm{S} 3}=(150 \mathrm{~m}-114 \mathrm{~m}) / 0.13 \mathrm{~s}=$ $276.92 \mathrm{~m} / \mathrm{s}$ and $\mathrm{V}_{\mathrm{S} 4}=(150 \mathrm{~m}-127.5 \mathrm{~m}) / 0.10 \mathrm{~s}=225 \mathrm{~m} / \mathrm{s}$. Thus, from SPEED node 1 has the largest velocity greater than $\mathrm{V}_{\text {req }}$ and will be chosen as the forwarder and so on.

As per THVR, node $\mathrm{S}$ will search among its two-hop neighbors $F_{2}^{+p}(S)$ i.e., nodes $(5,6,7,8,9,10)$ and calculate the velocity $\left(V_{x y \rightarrow z}\right)$ provided by each of the two-hop pairs by Equation. 9 ,

$$
\begin{aligned}
V_{S 3 \rightarrow 8}= & \frac{\operatorname{dist}(S, D)-\operatorname{dist}(8, D)}{\text { delay }_{S 3}+\operatorname{delay}_{38}} \\
= & \frac{150 m-90 m}{0.13 s+0.079 s} \\
& =287.08 m / s
\end{aligned}
$$

Similarly, the velocity provided by the two-hop pair:

$$
\begin{aligned}
& V_{S 1 \rightarrow 5}=(150 m-117 m) /(0.11 s+0.06 s)=235.7 m / s \\
& V_{S 1 \rightarrow 6}=(150 m-97.5 m) /(0.08 s+0.12 s)=262.5 m / s \\
& V_{S 2 \rightarrow 7}=(150 m-110 m) /(0.12 s+0.02 s)=285.7 m / s \\
& V_{S 3 \rightarrow 9}=(150 m-97.5 m) /(0.13 s+0.07 s)=262.5 m / s \\
& V_{S 4 \rightarrow 10}=(150 m-117 m) /(0.10 s+0.04 s)=235.7 m / s
\end{aligned}
$$

The velocity provided by $V_{S 3 \rightarrow 8}$ is greater than $V_{\text {req }}$ and is also the largest among the other two-hop pairs shown above. Therefore, node 3 will be chosen as the immediate forwarder. But, by RTLRR we also consider the PRR of the links while choosing the next forwarder, the PRR of link to node 2 is 0.9 and that to link 3 is 0.85 , hence node 2 will be chosen as the next hop candidate. If the packet arriving at node 2 has taken $0.13 \mathrm{~ms}$ to travel, then the new deadline to reach the destination will be $0.42 \mathrm{~s}$. The required velocity is updated at node 2 and the next forwarder is chosen based on this new value.

In RTLRR, by selecting a link that provides higher PRR, the protocol aids in increasing the probability of successful packet delivery to the forwarding node. In THVR, if a path from source to destination has a link with a poor packet reception ratio, then this may increase the DMR. By, selecting links providing greater PRR on the route, the throughput (amount of traffic successfully received by the destination) can be increased, obtain a lower DMR; augment the energy efficiency of the forwarding nodes due to lower number of collisions and retransmissions. Also, the two-hop neighborhood information incorporated with the dynamic velocity assignment policy will provide enhanced foresight to the sender in identifying the node pair that can provide the largest velocity towards the destination.

\section{Performance Evaluation}

To evaluate the proposed protocol, we carried out a simulation study using $n s-2$ [35]. The proposed protocol (RTLRR) is compared with THVR and SPEED. The simulation configuration consists of 200 nodes located in a $200 \mathrm{~m}^{2}$ area. Nodes are distributed following Poisson point process with a node density of 0.005 node $/ \mathrm{m}^{2}$. The source nodes are located in the region $(40 \mathrm{~m}, 40 \mathrm{~m})$ while the sink in the area $(200 \mathrm{~m}, 200 \mathrm{~m})$. The source generated a CBR flow of 1 packet/second with a packet size of 150 bytes.

The MAC layer, link quality and energy consumption parameters are set as per Mica2 Motes [36] with MPR400 radio as per THVR. Table 3 summarizes the simulation parameters. THVR and SPEED are QoS protocols and a comparison of DMR (Deadline Miss Ratio), ECPP (Energy Consumed Per Packet i.e., the total energy expended divided by the number of packets effectively transmitted), the packet average delay (mean of packet delay) and 
worst case delay (largest value sustained by the successful transmitted packet) are obtained.

In the first set of simulations, we consider 10 source nodes with varying deadlines from $100 \mathrm{~ms}$ to $700 \mathrm{~ms}$. In THVR, the weighting factor $\mathrm{C}$ is set at 0.9 to favor end-to-end delay performance, likewise in SPEED we assign $\mathrm{K}=10$ for shorter end-to-end delay. In the proposed protocol we set weighting factors $(\mathrm{A}, \mathrm{B}, \mathrm{C})$ at $(0.1,0.8,0.1)$. In each run, 500 packets are transmitted.

Table 3: Simulation Parameters

\begin{tabular}{|c|c|}
\hline Simulation Parameters Value & Value \\
\hline Number of nodes & 200 \\
\hline Simulation Topology & $200 \mathrm{~m}$ x $200 \mathrm{~m}$ \\
\hline Traffic & CBR \\
\hline Payload Size & 150 Bytes \\
\hline Transmission Range & $40 \mathrm{~m}$ \\
\hline Initial Battery Energy & 2.0 Joules \\
\hline Energy Consumed during Transmit & 0.0255 Joule \\
\hline Energy Consumed during Receive & 0.021 Joule \\
\hline Energy Consumed during Sleep & 0.000005 Joule \\
\hline Energy Consumed during Idle & 0.0096 Joule \\
\hline MAC Layer & 802.11 with DCF \\
\hline Propagation Model & Free Space \\
\hline Hello Period & 5 seconds \\
\hline PRR - WMEWMA Window & 30 \\
\hline PRR - WMEWMA Weight Factor $\alpha$ & 0.5 \\
\hline Delay - EWMA Weight Factor $\beta$ & 0.5 \\
\hline
\end{tabular}

Fig. 2 illustrates the efficiency of the RTLRR algorithm in reducing the DMR, the DMR characteristics of RTLRR and THVR are similar till a delay of $250 \mathrm{~ms}$ and the performance of RTLRR is better after that, eventually as the deadlines increase the DMRs converge to zero. In comparison, as shown in Fig. 2 THVR has a higher DMR, the initiative drop control has a slight negative effect on the DMR. In SPEED, when the deadline is stringent (less than $300 \mathrm{~ms}$ ), the SPEED protocol drops packets aggressively at lower deadlines, resulting in an overall higher DMR. Even, when the deadline is 700ms the DMR has not yet converged to zero. The two-hop based routing and dynamic velocity of the RTLRR algorithm is able to aggressively route more packets within the deadline to the sink node, also the protocol is able to select the reliable paths between the sources and the sink, hence it is observed that RTLRR has lower DMR than the others in general.

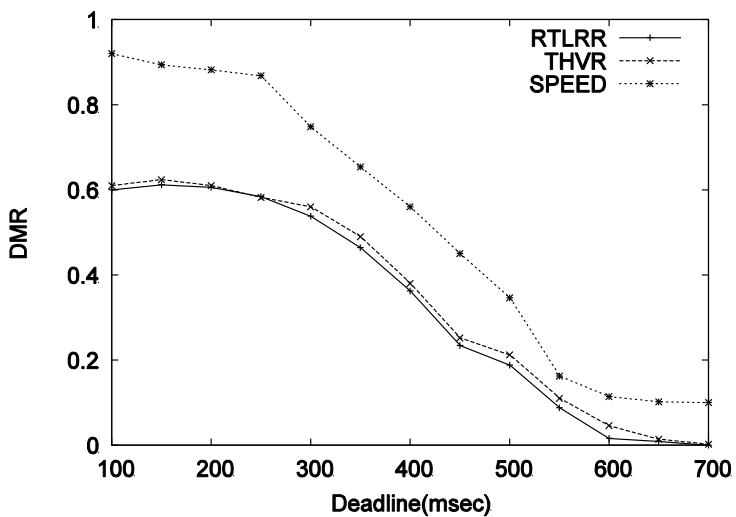

Fig. 2. DMR vs Deadline

As depicted in Fig. 3 the ECPP (energy consumption per packet) successfully transmitted, decreases as the deadline increases. The energy consumption has similar tendency in both RTLRR and THVR, but SPEED has a higher energy utilization. The slight variation of the RTLRR protocol is due to the link reliability incorporated in the route selection which may sometimes select a longer path to the destination resulting in higher energy utilization on some paths, but the dynamic velocity will minimize this effect. By, selecting links providing higher PRR on the route to the sink, the energy consumption of the forwarding nodes can be minimized, due to lower number of collisions and re-transmissions. Also, in the proposed protocol the link delay and packet delivery ratios are updated by piggybacking the information in ACK, this will help in reducing the number of feedback packets and hence reduce the total energy consumed. 


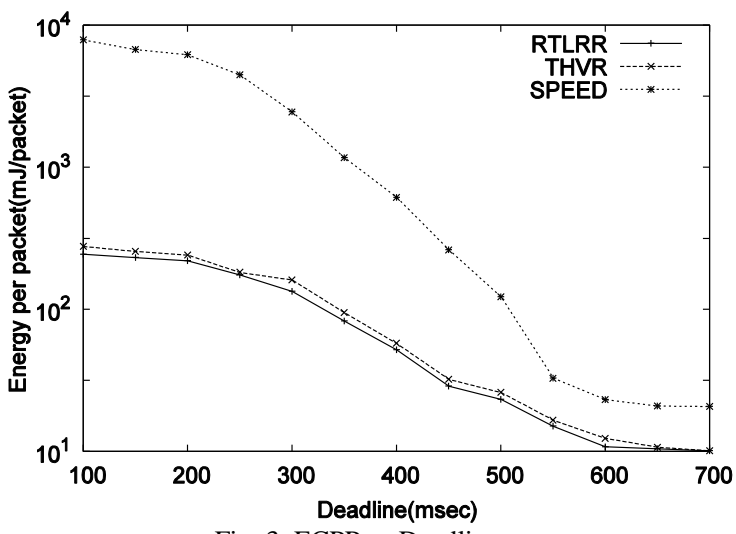

Fig. 3. ECPP vs Deadline

In THVR, the initiative drop control module, will drop the packet if it is near the source and cannot meet the required velocity, from the perspective of energy utilization. But, in the proposed protocol the packet will not be dropped since the dynamic velocity approach will aid in ensuring that the packet eventually meets the deadline, more packets will be forwarded to the destination and will improve the ECPP. Generally, RTLRR has a lower energy consumption level compared to the other protocols.

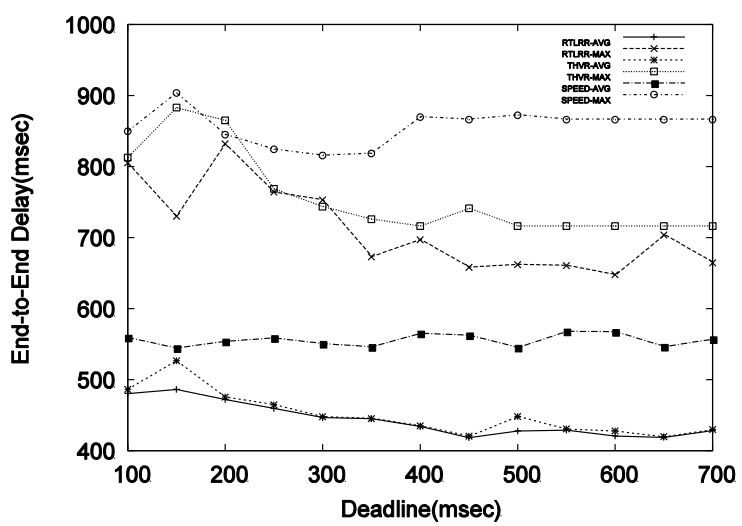

Fig. 4. Average and Worst case delay vs Deadline

Fig. 4 compares the packet end-to-end average and worst-case delays respectively. It is observed that THVR and RTLRR protocols have similar performance in the average end-to-end delay. The performance of RTLRR is better when the algorithms are compared in the worst-case delays. Performance of SPEED is poor in both the average and worst case delays. In RTLRR, paths from source to sink will be shorter due to the dynamic velocity, two-hop information and some variation in the delays because of link reliability, THVR will select path based only on two-hop routing information.

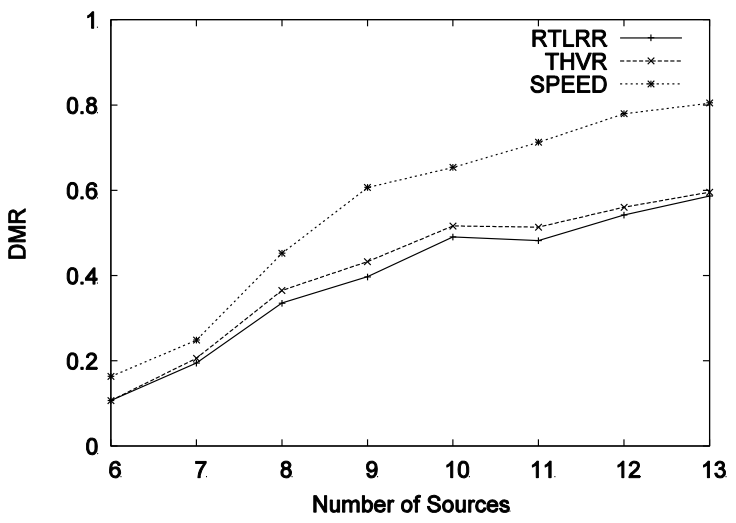

Fig. 5. DMR vs No. of Sources

Additionally, we examine the performance of the protocols under different loads. The number of sources is increased from 6 to 13; while the deadline requirement is fixed at $350 \mathrm{~ms}$. Each source generates a CBR flow of 1 packet/second with a packet size of 150 bytes.

From Fig. 5 and Fig. 6 it is observed that the DMR and ECPP plots ascend as the number of sources increase. 
The increase is resulted by the elevated channel busy probability, packet contentions at MAC and network congestion by the increased number of sources and resulting traffic. Examination of the plots illustrates that RTLRR protocol has lower DMR and also lower energy consumption per successfully transmitted packet.

Fig. 7 examines the packet end-to-end average and worst-case delays respectively. It is observed all the three protocols have similar performance in the average and worst case end-to-end delay, till the number of sources is 10. The performance of RTLRR is better because the algorithm is able to spread the routes to the destination, since greater number of source nodes help in finding links with more reliability in alternate paths and also provides better energy utilization. Simulation results reveal that there is a reduction in DMR, ECP and end-toend delays by the application of RTLRR algorithm. Overall link reliability, two-hop information and dynamic velocity enable the protocol to provide real time QoS support in WSNs.

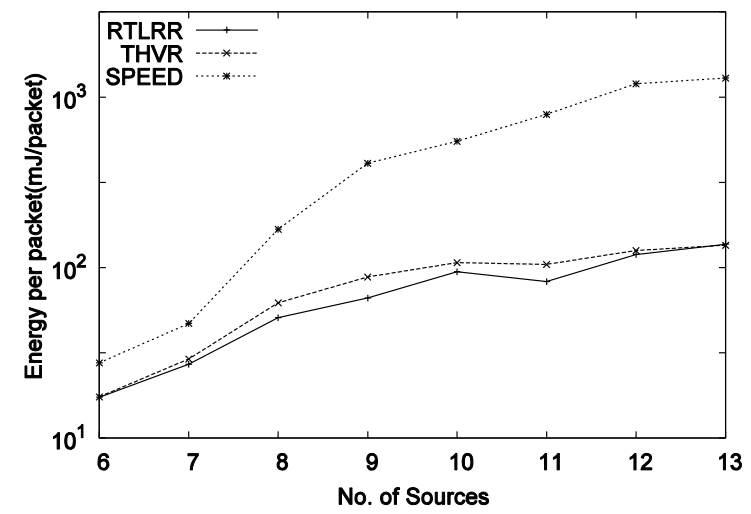

Fig. 6. ECPP vs No. of sources

Last, we study the performance of the residual energy cost function; the packet deadline is relaxed to a large value. Hence, when many nodes can provide the required velocity, a node that has high residual energy can be chosen as a forwarding node. This will result in uniform load balancing among the nodes of the network.

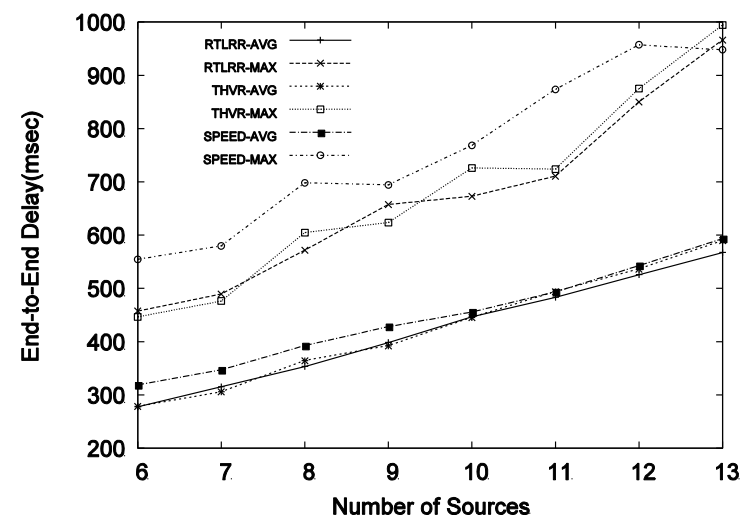

Fig. 7. Average and Worst case delay vs No. of sources

There are totally 200 nodes including 4 source nodes. The deadline is set to $600 \mathrm{~ms}$. In THVR, the weighting factor $\mathrm{C}$ is set at 0.7 to have a larger weight on residual energy, in the proposed protocol we set weighting factors $(\mathrm{A}, \mathrm{B}, \mathrm{C})$ to $(0.1,0.7,0.2)$. Fig. 8 and Fig. 9 show the node energy consumption distribution in THVR and RTLRR respectively after 200 runs.

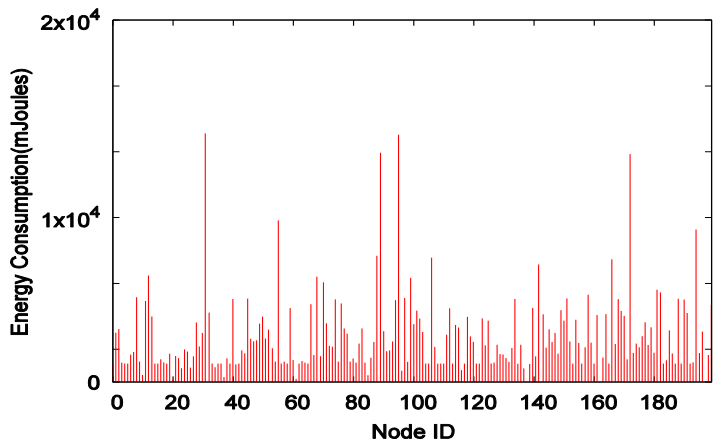

Fig. 8. Node energy consumption in THVR 
As observed in THVR, some nodes along the path from sources to sink are frequently chosen as forwarders and consume much more energy than the other, while in RTLRR only nodes close to the sources and sink consume relatively high energy. The latter is normal and inevitable especially as there may not be many optimal forwarding options near the sources and sink. Besides, by comparing Fig. 8 to Fig. 9, energy consumption in RTLRR is more evenly distributed among those between source and sink. The link reliability cost function will further aid to spread the routes to the destination compared to THVR. It can be observed that RTLRR will have a longer system lifetime due to the balancing.

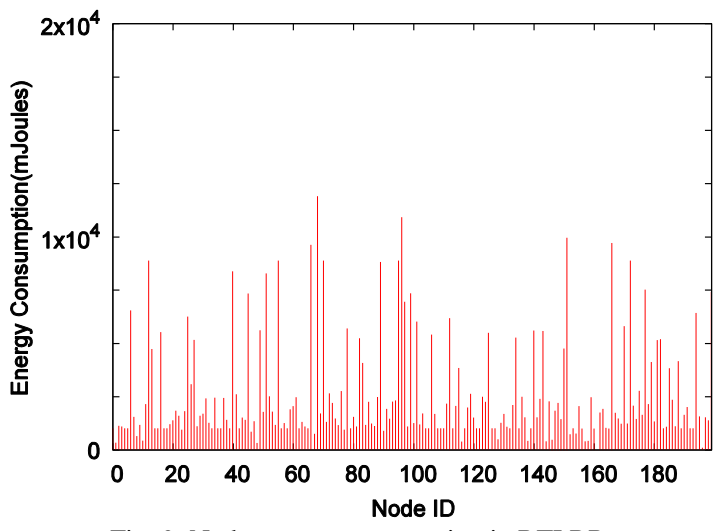

Fig. 9. Node energy consumption in RTLRR

\section{Conclusions}

In this paper, we propose a Real-Time Link Reliability Routing protocol for WSNs. Our proposed protocol is different from THVR, as it considers reliability and dynamic velocity that can be adjusted for each packet according to the required deadline. It balances the load only among nodes estimated to offer the required QoS. The RTLRR protocol is able to augment real-time delivery by an able integration of link reliability, twohop information and dynamic velocity. Future work can be carried out to support differentiated service and consider transmission power as a metric in forward node selection.

\section{REFERENCES}

[1] F. L. Lewis, D. J. Cook, S. K. Dasm and John Wiley. Wireless Sensor Networks, In Proc. Smart Environment Technologies, Protocols and Applications, New York, 2004, 1-18.

[2] Tian He, John A. Stankovic, Chenyang Lu, and Tarek F. Abdelzaher. A Spatiotemporal Protocol for Wireless Sensor Network, In IEEE Transactions on Parallel and Distributed Systems, 16(10), 2005, 995-1006.

[3] E. Felemban, C. G. Lee, and E. Ekici. MM-SPEED: Multipath Multi-Speed Protocol for QoS Quarantee of Reliability and Timeliness in Wireless Sensor Network, In IEEE Transactions on Mobile Computing, 5(6), 2006, 738-754.

[4] O. Chipara, Z. He, G. Xing, Q. Chen, X.Wang, C. Lu, J. Stankovic, and T. Abdelzaher. Real-Time Power-Aware Routing in Sensor Network, In Proc. IWQoS, 2006, 83-92.

[5] Y. Li, C. S. Chen and Y.-Q. Song, Enhancing Real-Time Delivery In Wireless Sensor Networks With Two-Hop Information. In IEEE Transactions On Industrial Informatics, 5(2), 2009, 113-122.

[6] J. Stankovic, T. Abdelzaher, C. Lu, L. Sha and J. Hou. Real-time Communication and Coordination in Embedded Sensor Networks, In Proc. IEEE, 91(7), 2003, 1002-1022.

[7] Karp and Kung. H. T. GPSR: Greedy Perimeter Stateless Routing for Wireless Networks. In Proc. 6th Annual International Conference on Mobile Computing and Networking (MobiCom), 2000, 243-254.

[8] Prosenjit Bose, Pat Morin, Ivan Stojmenovi, and Jorge Urrutia. Routing with Guaranteed Delivery in Ad hoc Wireless Networks, In Proc. of 3rd ACM Int. Workshop on Discrete Algorithms and Methods for Mobile Computing and Communications DIALM'99, $1999,48-55$.

[9] C. Lu, B. M. Blum, T. F. Abdelzaher, J. A. Stankovic, and T. He. RAP: A Real-Time Communication Architecture for Large-Scale Wireless Sensor Networks, In Proc. IEEE RTAS, September 2002.

[10] K. Sohrabi, J. Pottie. Protocols for Self-organization of A Wireless Sensor Network, In IEEE Personal Communications, 7(5), 2000, $16-27$.

[11] A. Mahapatra, K. Anand, and D.P. Agrawal. QoS and Energy Aware Routing for Real-TimeTraffic in Wireless Sensor Networks, In Computer Communications, 29(4), 2008, 437-445.

[12] D. Tran and H. Raghavendra. Routing with Congestion Awareness and Adaptivity in Mobile Ad Hoc Networks, In Proc. of IEEE WCNC, March 2005

[13] Y. Sankarasubramaniam, B. Akan, and I. F. Akyildiz. ESRT:Event-to-Sink Reliable Transport in Wireless Sensor Networks, In Proc. of ACM Mobihoc, 2003, 177-188

[14] X. Wu, B.J. dAuriol, J. Cho, and S. Lee. Optimal Routing in Sensor Networks for In-Home Health Monitoring with Multi Factor Considerations, In Proc. Sixth Annual IEEE International Conference Pervasive Computing and Comm. (PERCOM08), 2008, pages 720-725.

[15] T.L. Lim and M. Gurusamy. Energy Aware Geographical Routing and Topology Control to Improve Network Lifetime in Wireless Sensor Networks, In Proc. IEEE Intl Conf. Broad-band Networks (BROADNETS 05), 2005, 829-831.

[16] S. Wu and K.S. Candan. Power Aware Single and Multipath Geographic Routing in Sensor Networks, In Proc. IEEE Intl Conf. Broad-band Networks (BROADNETS 05), 5(7), 2007, 974-997. 
[17] C.-p. Li,W.-j. Hsu, B. Krishnamachari, and A. Helmy. A Local Metric for Geographic Routing with Power Control in Wireless Networks, In Proc. Second Ann. IEEE Conf. Sensor and Ad Hoc Comm and Networks (SECON), 2005, 229-239.

[18] K. Seada, M. Zuniga, A. Helmy, and B. Krishnamachari. Energy Efficient Forwarding Strategies for Geographic Routing in Lossy Wireless Sensor Networks, In Proc. ACM Sen-Sys, pages 108-121, 2004.

[19] M. M.-O.-R. Md.Abdur Razzaque, Muhammad Mahbub Alam and C. S.Hong. Multi-Constrained QoS Geographic Routing for Heterogeneous Traffic in Sensor Networks, In IEICE Transactions on Communications, 91B(8), 2008, $2589-2601$.

[20] K. Zeng, K. Ren, W. Lou, and P. J. Moran. Energy Aware Efficient Geographic Routing in Lossy Wireless Sensor Networks with Environmental Energy Supply, In Wireless Networks, 15(1):39-51, 2009.

[21] M. Chen, V. Leung, S. Mao, Y. Xiao, and I. Chlamtac. Hybrid Geographical Routing for Flexible Energy-Delay Trade-Offs, In IEEE Transactions on Vehicular Technology, 58(9), 2009, 4976-4988.

[22] T. L. Lim and M. Gurusamy. Energy Aware Geographical Routing and Topology Control to Improve Network Lifetime in Wireless Sensor Networks, In Proc. IEEE International Conference on Broadband Networks (BROAD-NETS05), 2005, 829-831.

[23] A. Sharif, V. Potdar and A.J.D Rathnayaka. Prioritizing Information for Achieving QoS Control in WSN, In Proc. IEEE International Conference on Advanced Information Networking and Applications, 2010, 835-842.

[24] M.E Rusli, R. Harris and A. Punchihewa. Markov Chain-based analytical model of Opportunistic Routing protocol for wireless sensor networks, In Proc. TENCON IEEE Region 10 Conference, 2010, pages 257-262.

[25] M. Koulali, A. Kobbane, M. El Koutbi and M. Azizi. QDGRP : A hybrid QoS Distributed Genetic routing protocol for Wireless Sensor Networks, In Proc. International Conference on Multimedia Computing and Systems, 2012, 47-52.

[26] Yunbo Wang, M.C Vuran and S. Goddard. Cross-Layer Analysis of the End-to-End Delay Distribution in Wireless Sensor Networks, In IEEE Transactions on Networking, 20(1), 2012, 305-318.

[27] S. Ehsan, B. Hamdaoui and M. Guizani. Radio and Medium Access Contention Aware Routing for Lifetime Maximization in Multichannel Sensor Networks, In IEEE Transactions on Wireless Communication, 11(9), 2012, 3058-3067.

[28] M. A. Spohn and J. J. Garcia-Luna-Aceves. Enhancing Broadcast Operations in Ad Hoc Networks with Two-Hop Connected Dominating Sets, In Proc. IEEE MASS, 2004, 543-545.

[29] G. Calinescu. Computing 2-hop Neighborhoods in Ad hoc Wireless Networks, in In Proc. AdHocNow, 2003, $175-186$.

[30] C. S. Chen, Y. Li, and Y.-Q. Song. An Exploration of Geographic Routing with K-hop Based Searching in Wireless Sensor Networks, In Proc. CHINACOM, 2008, 376-381.

[31] T. He, C. Huang, B.M. Blum, J.A. Stankovic, and T.F. Abdelzaher. Range-Free Localization and Its Impact on Large Scale Sensor Networks, In ACM Trans. Embedded Computer Systems, 4(4), 2000, 877-906.

[32] K. Zeng, K. Ren, W. Lou, and P.J. Moran. Energy Aware Efficient Geographic Routing in Lossy Wireless Sensor Networks with Environmental Energy Supply, In Wireless Networks, 15(1), 2009, 39-51.

[33] T. Roosta, M. Menzo, and S. Sastry. Probabilistic Geographical Routing Protocol for AdHoc and Sensor Networks, In Proc. Intl Workshop Wireless Ad-Hoc Networks (IWWAN), 2005.

[34] A. Woo and Culler. Evaluation of Efficient Link Reliability Estimators for Low-Power Wireless Networks, University of California, Tech. Rep., 2003

[35] NS-2, [Online]. Available: http://www.isi.edu/nsnam/ns/.

[36] Crossbow Motes, [Online]. Available: http://www.xbow.com. 